\title{
Erratum to: Erythropoietin Rescues Memory Impairment in a Rat Model of Chronic Cerebral Hypoperfusion via the EPO-R/JAK2/STAT5/PI3K/Akt/GSK-3 $\beta$ Pathway
}

\author{
Shengli Ma ${ }^{1} \cdot$ Juwu Chen $^{1} \cdot$ Chen $\mathrm{Chen}^{2} \cdot \mathrm{Na} \mathrm{Wei}^{3} \cdot$ Jingjing Xu ${ }^{3} \cdot$ Guohui Yang ${ }^{1}$. \\ Nan Wang ${ }^{1} \cdot$ Yu Meng ${ }^{1} \cdot$ Jia Ren $^{1} \cdot$ Zongchao Xu$^{1}$
}

Published online: 20 June 2017

(C) Springer Science+Business Media, LLC 2017

\section{Erratum to: Mol Neurobiol}

DOI 10.1007/s12035-017-0568-5

The original version of this article unfortunately contained a mistake in the Materials and Methods section.

In Page 2, the sentence "The rats were divided into the following four groups randomly: sham operation plus oral vehicle treatment (Sham), 2-VO surgery plus oral vehicle treatment (2VO), 2-VO surgery plus oral EPO (5000 IU/Kg, i.p., Shanghai Kehua Biopharmacy Inc. Shanghai, China) treatment (2VO+EPO), and sham operation plus EPO treatment (EPO)." should be corrected to read "The rats were divided into the following four groups randomly: sham operation plus $0.9 \%$ sodium choloride i.p. (Sham), 2-VO surgery plus $0.9 \%$ sodium choloride i.p. (2VO), 2-VO surgery plus EPO (5000 IU/Kg, i.p., Shanghai Kehua Biopharmacy Inc. Shanghai, China) treatment $(2 \mathrm{VO}+\mathrm{EPO})$, and sham operation plus EPO treatment (EPO).

The online version of the original article can be found at doi: http://dx.doi. org/10.1007/s12035-017-0568-5

Shengli Ma

ms104301617@163.com

1 Department of Emergency, Institute of Clinic Medicine, The First Affiliated Hospital of Zhengzhou University, No.1 Jian She Dong Avenue, Zhengzhou 450002, People's Republic of China

2 Department of Neurology, The First Affiliated Hospital of Zhengzhou University, No.1 Jian She Dong Avenue, Zhengzhou 450002, People's Republic of China

3 Department of Pathology, The First Affiliated Hospital of Zhengzhou University, No.1 Jian She Dong Avenue, Zhengzhou 450002,

People's Republic of China 